Revista de Psicología Vol. 37 (1), 2019 (ISSN 0254-9247)

\title{
Uso de TIC en prácticas pedagógicas de docentes de la Facultad de Psicología de una universidad pública argentina
}

\author{
Ana Borgobello ${ }^{1,}$ Melisa Madolesi ${ }^{2}$, Andrea Espinosa $^{3}$, Mariana Sartori ${ }^{4}$ \\ IRICE-CONICET $T^{14}$, Universidad Nacional de Rosario-Argentina ${ }^{1234}$
}

Se describen y construyen perfiles de docentes universitarios de una universidad pública argentina respecto al uso de TIC en sus prácticas pedagógicas a partir de la aplicación de un cuestionario online sobre características personales y académicas, experiencias, formación y formas mediadas de comunicación con los estudiantes. Se hicieron análisis descriptivo, multivariado y de contenido. Los resultados muestran tres perfiles con posicionamientos diferenciales en aspectos tecnopedagógicos determinados por las preferencias y experiencias respecto al uso de Facebook, WhatsApp y Plataforma virtual. En las narraciones se observan posturas críticas diferenciales de acuerdo al perfil. La interpretación y discusión de los datos fueron contextualizados en un escenario poco favorecedor hacia el uso de TIC.

Palabras clave: comunicación mediada por ordenador, entornos de aprendizaje, comunicación electrónica, redes sociales en línea, aulas virtuales.

Use of ICT in pedagogical practices of teachers of the Faculty of Psychology of a public university in Argentina

This study aims to create and describe profiles of public university professors in Argentina based on their use of ICTs in pedagogical practices. The study used an online questionnaire that measures personal and academic characteristics, experiences, training and mediated forms of communication with students. Descriptive, multivariate and content analyses were performed. The results show three profiles with differential positions in techno-

1 Doctora en Psicología. Especialista en entornos virtuales en contextos de enseñanza y aprendizaje de nivel universitario. Docente de Metodologías de la Investigación en Psicología. Dirección postal: 27 de Febrero 210bis, Rosario, Argentina. Contacto: borgobello@iriceconicet.gov.ar

2 Docente de Psicología en la Universidad Nacional de Rosario. Dirección postal: Riobamba 250bis, Rosario, Argentina. Contacto: melisamandolesi@unr.edu.ar.

3 Psicóloga. Especialista en Metodología de la Investigación Científica. Docente de distintas materias de metodologías de la investigación. Dirección postal: Riobamba 250bis, Rosario, Santa Fe, Argentina. andrea_espinosa01@yahoo.com.ar

4 Psicóloga. Becaria doctoral Agencia Nacional de Promoción Científica y Tecnológica. Dirección postal: 27 de Febrero 210bis (entrada por Ocampo y Esmeralda), Rosario, Santa Fe, Argentina.sartori@irice-conicet.gov.ar 
pedagogical aspects determined by the use of Facebook, WhatsApp and Virtual Platform. In the narratives, differential critical positions were observed according to each profile. The interpretation and discussion of results were contextualized ina scenario that is not favorable towards the use of ICT.

Keywords: computer mediated communication, learning environments, electronic communication, online social networks, virtual classrooms.

\section{Uso das TIC nas práticas pedagógicas de professores da Faculdade de Psicologia de uma universidade pública da Argentina}

Os perfis de docentes e professores universitários foram descritos sobre o uso das TIC nas práticas pedagógicas. $\mathrm{O}$ estudo baseou-se nas respostas a um questionário on-line que solicitava características e experiências acadêmicas, experiências, treinamento e formas de comunicação mediadas com os alunos. Foram realizadas análise descritiva, multivariada e de conteúdo. Os resultados mostraram três perfis com posiçóes diferenciais em aspectos tecno-pedagógicos determinados pelo uso do Facebook, WhatsApp e Plataforma Virtual. Nas narrativas, as posiçôes críticas observadas foram diferenciais de acordo com cada perfil. A interpretaçáo e discussáo de resultados foram contextualizadas em um cenário náo auspicioso para o uso das TIC.

Palavras chave: comunicação através de computadores, ambientes de aprendizagem, comunicação eletrônica, redes sociais on-line, salas de aula virtuais

Utilisation des TIC dans les pratiques pédagogiques des professeurs de la faculté de psychologie d'une université publique d'Argentine

Les profils des professeurs d'université sont décrits et construits en fonction de l'utilisation des TIC dans leurs pratiques pédagogiques, basée sur l'application d'un questionnaire en ligne sur les caractéristiques personnelles et académiques, les expériences, la formation et les formes médiatiques de communication avec les étudiants. Une analyse descriptive, multivariée et de contenu a été faite. Les résultats montrent trois profils avec des positions différentielles dans les aspects technopédagogiques déterminés par l'utilisation de Facebook, WhatsApp et Virtual Platform. Dans les discours, les positions critiques différentielles sont observées en fonction du profil. L'interprétation et la discussion sont contextualisées dans un scénario peu d'espoir pour l'utilisation des TIC.

Mots-clés: communication par ordinateurs, environnements d'apprentissage, communication électronique, réseaux sociaux en ligne, salles de classe virtuelles 
Este artículo se propone socializar inquietudes y experiencias acerca de la formación docente y el uso de tecnologías de la información y la comunicación (TIC) en el nivel superior. Por tanto, está dirigido a quienes compartan estas preocupaciones, docentes e investigadores en psicología de la educación y ciencias afines, entendiendo que toda práctica docente está atravesada y construida en contextos sociales específicos.

Esta manera de entender la educación formal se basa en las experiencias del equipo en prácticas educativas, en investigación y formación de docentes en el uso de la plataforma virtual Moodle. El enfoque que sostenemos postula la centralidad del docente para la innovación educativa (Maggio, 2016). En este sentido, no se trata de enumerar la mera presencia de TIC en la comunicación con los estudiantes sino de comprender las relaciones y usos de TIC en interacción en proyectos pedagógicos con visiones críticas. A partir de estas preocupaciones y experiencias, entendemos la necesidad de trabajar con datos empíricos, retomando aportes teóricos, es decir, construyendo conocimientos situados desde posturas filosóficas, teóricas y análisis de datos en forma dinámica y entrelazada.

Las TIC, eje del presente trabajo, han irrumpido en el ámbito de la vida cotidiana, incluyendo la educación formal. No obstante, los modelos clásicos no han desaparecido (García-Aretio, 2017). Así, Turpo (2014) postula que las TIC en educación implican un paradigma de renovación continua. La pedagogía y la tecnología debieran ser entendidas en continuidad y no como antagónicas dándole un sentido tecnopedagógico a la convergencia. González-Fernández (2018), además, destaca la importancia de contemplar necesidades contextuales en las estrategias tecnopedagógicas. Sostiene que los aspectos contextuales como el contenido disciplinar, las competencias del grupo en términos de autogestión del aprendizaje, habilidades tecnológicas, capacidad de comunicación utilizando Internet, entre otros, determinarán la efectiva integración tecnológica. 
Lipsman (2016), por su parte, plantea que los propósitos pedagógicos que subyacen a las propuestas que incorporan TIC en la enseñanza, invitan a los docentes a ser protagonistas de la construcción de las actividades que proponen a sus estudiantes. Para lo cual, según la autora, debieran disponer de momentos de exploración con los recursos tecnológicos, aunque estos momentos no existen hasta ahora. Aun así, Turpo (2010) sostiene que en la implementación de TIC en educación, la mayoría de los docentes asume roles tanto de contenidista como de tutor, es decir, diseñan y supervisan las tareas de aprendizaje de los estudiantes en su conjunto. Sin embargo, según observa Pierella (2014), en el contexto local los estudiantes critican la falta de adecuación de los docentes a las nuevas tecnologías.

Las redes sociales son TIC inmersas en la vida cotidiana, han transformado y están transformando las formas de relacionarnos y comunicarnos. Sin embargo, Martín y Tourón (2017) destacan que las universidades no han sido suficientemente ágiles para integrarlas en las dinámicas pedagógicas de maneras realmente efectivas.

Las formas de enseñar y aprender soportadas por sistemas digitales se van distribuyendo en todos los rincones del planeta, presentando formatos diversos según las visiones pedagógicas, psicológicas, filosóficas, sociológicas, políticas y en relación a las disponibilidades económicas y tecnológicas. En estos cambios, García-Aretio (2017) se pregunta si realmente los estudiantes han cambiado de manera radical sus formas de adquirir conocimiento y, de ser así, si las formas de enseñar deberían hacer lo propio.

Turpo (2014) plantea que nos encontramos ante un salto inevitable signado por la convergencia que hace imperceptibles las fronteras entre la presencialidad y la virtualidad generando nuevas síntesis pedagógicas y tecnológicas percibiéndose como intercambio continuo. Así, es frecuente encontrar estudiantes en clases presenciales revisando desde el teléfono celular el contenido de la plataforma virtual, aún en condiciones de bajísima conectividad como las del entorno educativo en el que se desarrolla la investigación aquí presentada. Boelens, DeWever y Voet (2017) sostienen que, entre los desafíos que propone 
Uso de TIC en prácticas pedagógicas de docentes de la Facultad de Psicología... / Borgobello et al.

la incorporación de la virtualidad combinada con los espacios tradicionales de enseñanza, se encuentran el aumento de flexibilidad e interacción que posibilitan facilitar los aprendizajes de los estudiantes.

La dualidad es propia de la enseñanza blended learning (BL) que se remonta a unos 20 años atrás (Turpo, 2014). Como modalidad formativa, tiene connotaciones y denominaciones diversas, pero con una configuración común básica: combina presencialidad y virtualidad, por lo que hibridiza, integra y mezcla dos modalidades educativas distintas. BL refiere a cómo son presentados los contenidos a los estudiantes. Cuando un conjunto de contenidos es diseñado, resulta relevante que el mismo sea presentado de tantas maneras distintas como sea posible dando así lugar a diferentes estrategias de enseñanza y aprendizaje (Eagleton, 2017). La modalidad BL es adaptable a pregrado y posgrado, versátil y factible. Presenta soportes tecnológicos variados que dependen en gran medida del contexto de pertenencia del proyecto educativo, siendo los más utilizados las plataformas electrónicas que ofrecen ambientes colaborativos para los procesos de enseñanza y aprendizaje (Turpo, 2010).

En este sentido, Villar (2017) sostiene que la universidad argentina asume el desafío de ir incorporando TIC paulatinamente, encaminándose hacia el cursado bimodal (BL). Subraya que los espacios físicos presenciales de encuentros pedagógicos subsistirán generando trayectorias pedagógicas alternativas y diferenciales en escenarios propios de una universidad masiva. La cantidad de alumnos en el contexto de la universidad pública argentina resulta una problemática institucional a la que es necesario dar respuesta configurando nuevas estrategias pedagógicas. En este sentido, “... los espacios físicos muchas veces resultan insuficientes y los entornos virtuales se van convirtiendo en una alternativa" (Lipsman, 2016, p. 145).

En plena relación con lo aquí expuesto, una serie de estudios llevados a cabo en la Facultad de Psicología de la Universidad de Buenos Aires (UBA), describieron y analizaron tanto los recursos disponibles y utilizados por las cátedras, como la percepción e implementación por parte de los docentes (Fernández-Zalazar, Jofre, Pisani \& Ciacciulli, 2015; Fernández-Zalazar \& Neri, 2015; Fernández-Zalazar, Jofre \& 
Soto, 2016). Encontraron que se trata aún de algo incipiente y basado en un uso instrumental-mercantil de las TIC, sin una reflexión crítica de las concepciones pedagógicas que subyacen a estas prácticas. Asimismo, hallaron escasa transferencia de habilidades y estrategias en el uso de TIC de lo cotidiano a lo educativo. Esto se ve reflejado en mayor uso de recursos digitales impulsados por el mercado como las redes sociales y la escasa implementación de entornos de aprendizaje. Así por ejemplo, en lo que respecta al uso de entornos de aprendizaje virtuales, solo cerca de un $6 \%$ de las materias lo implementaban y los foros de discusión solo eran utilizados por aproximadamente el $3 \%$ de las cátedras, siendo más frecuente la utilización de Facebook (35\%) y página web institucional (64\%). Cabe destacar que el uso estaba destinado a repositorio de información o reproducción de contenidos detallados en los programas de las asignaturas. Es decir, el uso de TIC destinado a la interacción explícita entre docentes y estudiantes era poco frecuente, utilizándose fundamentalmente como un "depósito de archivos" para que los estudiantes descarguen, uso similar al que en otros tiempos cumplían las fotocopiadoras.

Así, si bien la mayoría de los docentes (89\%) consideraba útil la incorporación de las TIC en educación, y la mitad de los mismos usaban las TIC como recurso para el dictado de clases, se trata de usos tradicionales, como proyector, cañón y presentaciones. Además, un $60 \%$ de los docentes refirió no haber recibido ningún tipo de formación en implementación de TIC en educación y, quienes habían recibido formación, tuvo lugar en un contexto informal o de modo autodidacta (FernándezZalazar et al., 2015, 2016; Fernández-Zalazar \& Neri, 2015).

En síntesis, se observa en la literatura un modelo tradicional de enseñanza que incorpora insuficientemente el potencial innovador que pudieran aportar las TIC a la docencia universitaria.

\section{Contextualización del estudio}

Respecto al contexto universitario local, el uso de espacios virtuales puede considerarse escaso, predominando la enseñanza tradicional con 
clases magistrales (Borgobello, Sartori \& Roselli, 2016). En este sentido, Copertari, Sgreccia y Segura (2011) sostienen que en los últimos tiempos hubo cierto avance en la implementación de sistemas bimodales y virtuales en el contexto universitario local. Desde 2008, la universidad tiene a disposición de la comunidad educativa el campus virtual con plataforma Moodle instalada. Actualmente, las unidades académicas que integran la institución desarrollan proyectos de pregrado, grado, posgrado y abiertos a la comunidad en el espacio denominado Comunidades del campus virtual. A pesar de ello, Copertari et al. (2015) aseguran que aún no se ha logrado una suficiente consolidación de prácticas con TIC. Asimismo, hipotetizan que desde las políticas educativas, como así también desde la cultura académica predominante, no se vislumbra la potencialidad de la modalidad blended learning para prácticas pedagógicas universitarias. Las experiencias docentes reportadas en esta universidad denotan esfuerzos diferentes, aunque aún dispersos en la implementación de TIC, mostrando la necesidad de seguir aportando al entendimiento, divulgación y afianzamiento de este tipo de espacios (Borgobello et al., 2016).

El estudio que aquí se presenta forma parte de una línea de investigación más amplia que se ocupa de la incorporación de TIC en el contexto universitario local, especialmente, del llamado blended learning (Borgobello \& Roselli, 2016; Borgobello et al., 2016; Borgobello, Sartori \& Sanjurjo, 2018). Desde una perspectiva multimétodos, nuestra forma de trabajo implica articular estrategias, métodos y técnicas provenientes de enfoques concebidos como tradicionalmente opuestos, de modo tal de poder captar y estudiar al fenómeno considerando su complejidad. Así, realizamos una combinación de miradas cualitativas y cuantitativas, observaciones, cuasi-experimentos, entrevistas en profundidad, cuestionarios, entre otros. En esta ocasión, presentamos los datos provenientes de la aplicación de un cuestionario autoadministrado con modalidad online a docentes universitarios cuyo propósito fue indagar sobre el uso de TIC en el ámbito pedagógico.

Este estudio fue realizado en una Facultad de Psicología de una universidad pública argentina. En la misma se dictan las carreras de Psicología 
y Profesorado en Psicología que comparten numerosas materias, siendo la carrera que otorga el título de Psicólogo la que posee la mayor cantidad de estudiantes, cátedras y docentes. Debido a avatares históricos, políticos y sociales del país, esta institución sostiene la formación en una tradición clínica y psicoanalítica, siendo una de las pocas Facultades de Psicología del país que otorgan título de Psicólogo — profesional- y no de Licenciado en Psicología (Klappenbach, 2015). Asimismo, el perfil típico docente es un profesional que trabaja en clínica — generalmente privada - como fuente principal de ingresos y en docencia con dedicación simple, es decir, suelen tener una sola clase semanal a cargo.

Actualmente, la Facultad se encuentra atravesando un proceso de adecuación del Plan de Estudios de la Carrera de Psicología a los requerimientos de la Comisión Nacional de Evaluación y Acreditación Universitaria (CoNEAU). A pesar de la abierta oposición de la comunidad universitaria en su conjunto a los lineamientos generales de la Ley de Educación Superior que sirve como marco legal a la CoNEAU, el proceso de adecuación se inició en 2011. Luego de diversas instancias de evaluación, se aprobó en 2014 un Plan de Estudios de transición que es el que se encuentra actualmente vigente.

El Plan de transición consta de 35 materias obligatorias y el Profesorado de 25, ambas carreras comparten la mayoría de las materias. Las asignaturas se encuentran distribuidas en ocho Departamentos en los que se organiza la docencia: Primer Año; del área Social Comunitaria; Psicoanálisis; Estudios Interdisciplinarios: Biología, Psicología y Cultura; del área Histórica Epistemológica; del área Socio Educativa; del área Investigaciones; y Clínicas. Cabe señalar que, si bien el cambio curricular implicó dar nuevas denominaciones a la mayoría de las asignaturas y modificar la estructura de la malla curricular, existe consenso entre los docentes en percibir las modificaciones, incluso en lo que hace a los contenidos, como cambios poco profundos en las prácticas pedagógicas habituales de la enseñanza y aprendizaje de la Facultad.

La mayor parte de las asignaturas tienen dos instancias de cursado. Habitualmente, las comisiones de trabajos prácticos — a cargo de JTP (Jefe de Trabajos Prácticos) y Auxiliares de Primera — son obligatorios 
mientras que las clases teóricas magistrales son optativas. La cantidad de docentes de cada cátedra, así como sus dedicaciones son disímiles, dependiendo esta distribución de numerosas circunstancias históricas y epistémicas. Esta situación influye en forma directa en la distribución de estudiantes que cursan en cada comisión de trabajos prácticos y que asisten a las clases teóricas magistrales. Se trata de un contexto altamente politizado y flexible en las estrategias de trabajo — entre las que se incluye el uso o no de plataforma virtual, uno de los ejes del presente estudio- que permite a los docentes priorizar temas que consideren adecuados enfatizando las perspectivas con las que más acuerden. Si bien, como expresa Lipsman (2016), la tendencia mundial, aun en educación, es la obsolescencia, es decir, a renovar objetos — prácticas, teorías, conceptos - por el hecho de la existencia de otros nuevos, la universidad argentina parece esquivar de alguna manera esta tendencia.

Este estudio se enmarca específicamente en un Proyecto de Investigación y Desarrollo "Los entornos virtuales en la enseñanza universitaria”, acreditado por una Facultad de Psicología dependiente de una universidad pública nacional de Argentina. La investigación se centró en explorar concepciones, creencias y evaluaciones de docentes referentes a la docencia universitaria y a los usos reales y posibles de entornos virtuales.

El objetivo específico de este estudio fue explorar las características y opiniones de los docentes universitarios de una Facultad de Psicología pública respecto del uso de TIC en las prácticas pedagógicas. A partir de ese objetivo, se construyó un cuestionario autoadministrado dirigido a docentes de una Facultad de Psicología exclusivamente. A continuación, enunciamos algunas de las preguntas de investigación que guiaron la construcción del instrumento y el análisis de los datos:

- ¿Qué características tienen los docentes universitarios que utilizan TIC en sus prácticas pedagógicas?

- ¿Qué conocimiento y uso de TIC en prácticas pedagógicas tienen los y las docentes de la Facultad?

- ¿Qué críticas pedagógicas, instruccionales e institucionales hacen en relación al uso de TIC? 


\section{Método}

\section{Participantes}

La Facultad de Psicología en la que se realizó el estudio cuenta con una planta de 452 docentes. A través del cuestionario administrado online obtuvimos 63 respuestas válidas, es decir, un 14\% de la población total. Este porcentaje puede considerarse adecuado debido a las características globales de la institución con la que se trabajó.

Respecto a las edades de los docentes que respondieron, 18 (29\%) tenían entre 25 y 39 años, 28 (44\%) entre 40 y 54 años y 17 (27\%) eran mayores de 55 ańos. La antigüedad en docencia declarada — desde el comienzo como auxiliares alumnos en adelante- fue desde cuatro a 38 años (con una media de 20 y un desvío estándar amplio de 8,9). Nueve de los participantes (14\%) tenían al momento de responder hasta diez ańos de antigüedad, 23 (37\%) entre 11 y 20 ańos, 20 (32\%) entre 21 y 30 ańos y los 11 restantes (17\%) más de 30 años.

El $81 \%$ de los respondientes (51 sujetos) tienen como título de grado Psicólogas/os, el resto se encuentran distribuidos con escasa frecuencia entre Lic. en Filosofía, en Letras, en Ciencias de la Educación, en Ciencia Política, en Psicopedagogía, en Fonoaudiología y Médicos/as.

La mayor parte de los respondientes, 32, poseen un título de posgrado $(51 \%)$ y solo $8(13 \%)$ no poseen ningún título de posgrado ni se encuentran cursando una carrera de especialización, maestría o doctorado (Tabla 1).

Algo más de la mitad de los respondientes, 33 (52\%), eran Jefes de Trabajos Prácticos, 19 (30\%) Adjuntos y Asociados y 11 (18\%) Titulares, es decir, encontramos respuesta en docentes con diferentes jerarquías dentro de la estructura universitaria. En cuanto a la dedicación a la docencia, 19 tenían al momento de completar el cuestionario dedicación simple (30\%), 37 semiexclusiva (59\%) y 7 exclusiva (11\%). 
Uso de TIC en prácticas pedagógicas de docentes de la Facultad de Psicología... / Borgobello et al.

\section{Tabla 1}

Título máximo de posgrado alcanzado o en curso

\begin{tabular}{lcc}
\hline Situación de posgrado & $\mathrm{f}$ & $\%$ \\
\hline No está cursando un posgrado & 8 & 13 \\
Está cursando una Especialización & 5 & 8 \\
Es Especialista & 6 & 10 \\
Es cursando una Maestría & 7 & 11 \\
Es Magíster & 9 & 14 \\
Está cursando el Doctorado & 11 & 18 \\
Es Doctor/a en... & 17 & 27 \\
\hline
\end{tabular}

\section{Tabla 2}

Departamentols en el que se inscribe la materia en la que el docente dicta clases de acuerdo al Plan de Estudios vigente

\begin{tabular}{lccccc}
\hline Departamento & f & $\%$ & $\begin{array}{c}\text { Docentes por } \\
\text { departamento }\end{array}$ & $\begin{array}{c}\text { \% relativo a la cantidad } \\
\text { de docentes del } \\
\text { Departamento }\end{array}$ \\
\hline Primer Año & 17 & 27 & 80 & 21 \\
Del Área Investigaciones & 11 & 18 & 29 & 38 \\
Del Área Socio-Educativa & 9 & 14 & 31 & 29 \\
Del Área Estudios Interdisciplinarios & 9 & 14 & 49 & 18 \\
Del Área de Clínicas & 9 & 14 & 70 & 13 \\
Del Área Social y Comunitaria & 7 & 11 & 102 & 6 \\
Del Área de Psicoanálisis & 5 & 8 & 79 & 2 \\
Del Área Histórico-Epistemológica & 2 & 3 & 88 & 6 \\
\hline
\end{tabular}

Nota 1. Debido a que algunos docentes dan clases en diferentes materias, la sumatoria no corresponde a la $\mathrm{N}$.

Nota 2. Los números de la columna "Docentes por departamento" corresponden a personas, los cargos podían ser de dedicación simple, semiexclusiva y exclusiva. 
En cuanto a los Departamentos de organización de la docencia (Tabla 2), la mayor parte de quienes respondieron, eran de Primer Año - Departamento conformado por materias de diferentes características epistémicas que reúnen contenidos introductorios a diferentes disciplinas. Del Área de Investigaciones obtuvimos 11 respuestas (18\%) que, siendo un Departamento integrado por pocos docentes, es una frecuencia elevada. La menor frecuencia de respuestas se obtuvo con relación al Departamento del Área Histórico-Epistemológica.

\section{Tabla 3}

Años en los que dicta clases cada docente

\begin{tabular}{lcc}
\hline Año/Profesorado & $\mathrm{f}$ & $\%$ \\
\hline Primer Año & 17 & 27 \\
Segundo Año & 10 & 16 \\
Tercer Año & 5 & 8 \\
Cuarto Año & 12 & 19 \\
Quinto Año & 10 & 16 \\
Sexto Año & 15 & 24 \\
Profesorado en Psicología & 3 & 5 \\
\hline
\end{tabular}

Nota 1. Debido a que algunos docentes dan clases en diferentes materias, la sumatoria de la tabla no corresponde a la $\mathrm{N}$.

Nota 2. Dado que el Profesorado puede cursarse en diferentes momentos y es una carrera diferente a la de Psicología, fue consignado por separado en la tabla.

En cuanto a la distribución de los docentes en años de cursado (Tabla 3) en los que se dictan clases, si bien se encuentran representados todos los años, la frecuencia de respondientes de sexto año es alta en relación a que allí trabajan presumiblemente la menor cantidad de docentes de la Carrera. La cantidad de alumnos con los que trabajan los docentes que respondieron oscila entre 15 y 550 , variando de acuerdo a la situación de la materia en la malla curricular del Plan de transición —algunas asignaturas cambiaron de ańo de cursado-, si dictan solo teóricos, solo prácticos o ambos. 
Uso de TIC en prácticas pedagógicas de docentes de la Facultad de Psicología... / Borgobello et al.

\section{Medición}

Las mediciones, y a partir de ellas los resultados que se presentan, se basan en una postura teórica paradigmática propia de metodologías cualitativas. Desde allí se combinan datos cuantitativos (numéricos) y cualitativos en un modo de hacer investigación coherente entre metodología y diseño (Twining, Heller, Nussbaum \& Tsai, 2017). En proyectos en los que el contexto tiene un peso específico propio, como este caso, el uso de preguntas abiertas permite recabar argumentaciones e incorporar aspectos cualitativos y contextuales a los análisis (Pozzo, Borgobello \& Pierella, 2018), razón por la cual el cuestionario aplicado fue construido con preguntas cerradas y abiertas.

La construcción del instrumento (véase Apéndice 1) se basó en las siguientes variables en estudio: características sociodemográficas y laborales, intereses disciplinares, conocimientos, experiencias y usos de TIC. Para caracterizar a los encuestados, se preguntó edad, título de grado y posgrado — si lo tuviese-, área de interés disciplinar, experiencia como alumno con algún cursado virtual y formación en TIC para la docencia. Se sumó a la indagación la situación de docencia: tipo de cargo, cantidad de alumnos, años de antigüedad y tipo de dedicación, Departamento y año en el que dictaba clases y cantidad de clases semanales.

En cuanto a conocimiento y experiencias con TIC, se exploró: si conocían qué son los espacios, plataformas o entornos virtuales y que los mismos se encuentran a disposición de las y los docentes desde 2001; acerca del uso de correo electrónico, aula virtual, WhatsApp y Facebook con los estudiantes; y si otro/s miembro/s de la cátedra en la que trabaja usa/n plataforma virtual. Adicionalmente, dado que como parte del proyecto se dictó un taller gratuito para que los docentes puedan iniciarse en el uso de Moodle, se preguntó si les interesaba participar. Finalmente, se construyó una pregunta abierta en la que los docentes pudieran expresar opiniones sobre el instrumento o sobre el tema indagado.

En cuanto al cálculo de la fiabilidad del cuestionario construido, se obtuvo un Alfa de Cronbach global de .68 para el conjunto de las 7 preguntas referidas a TIC de las que consta el cuestionario (preguntas 15 a 21 del Apéndice 1). 


\section{Procedimiento}

La construcción del cuestionario en su versión implementada contempló una serie de pruebas piloto y revisión de expertos. La distribución se realizó por intermedio de la Secretaría de Ciencia y Tecnología a través de correo electrónico a una base de datos compuesta por docentes de la casa. Las respuestas fueron digitales y completamente anónimas. Estas características permitieron que la recolección de datos se haga sin intermediación personal de los investigadores, evidenciando un origen confiable para los respondientes. Una ventaja de este tipo de instrumentos es la garantía de anonimato — reforzada por la masividad y el soporte digital - dando lugar a la expresión abierta en temas delicados (Pozzo et al., 2018).

El consentimiento informado con los datos institucionales, del proyecto y de los investigadores responsables del mismo, se encontraban disponibles en la sección superior del instrumento (véase Apéndice 1).

\section{Análisis de datos}

Se realizó un análisis multivariado de los datos de correspondencias múltiples con clasificación de coordenadas factoriales y selección de variables activas e ilustrativas (Moscoloni, 2005). Se utilizó el software Système Portable Pour L'analyse des Données (SPAD). El mismo utiliza variables activas elegidas por los investigadores, siendo aquellas que participan directamente en las fases del cálculo, especialmente en la formación de los factores. Por otro lado, el software permite la selección de variables ilustrativas que son tomadas por el programa en ciertas fases del cálculo, pero que se utilizan para establecer criterios de comparación (Parra-Olivares, 1996).

Las variables activas fueron dicotómicas y referían a: cursado con entorno virtual como estudiante, uso de Facebook, WhatsApp y plataforma virtual con los estudiantes en situación de docente. Las otras variables descritas arriba fueron cargadas como variables ilustrativas. Se describen los dos factores vectoriales y la agrupación de los casos en tres clusters, grupos o perfiles. Adicionalmente, se transcriben las respuestas 
abiertas al cuestionario de los diez casos más representativos de cada grupo. Finalmente, se analizó el contenido de las respuestas abiertas de los casos más representativos de cada cluster en función de las críticas pedagógicas, instruccionales e institucionales que los docentes hicieron en relación al uso de TIC.

Un aspecto central para la construcción de los perfiles desde el análisis de correspondencias múltiples con clasificación de coordenadas factoriales, siguiendo a Aluja-Banet y Morineau (1999), es la selección de variables activas que determinan la construcción de los ejes en los planos factoriales. A su vez, las variables ilustrativas conforman elementos necesarios para la interpretación de los datos. Por esa razón, se detallan al inicio del apartado correspondiente los resultados descriptivos de las principales variables elegidas como activas e ilustrativas relativas al conocimiento, usos de TIC y opiniones de los docentes.

\section{Resultados}

\section{Conocimiento, usos de TIC y opiniones de los docentes}

A continuación se detallan los resultados descriptivos acerca de los usos de TIC en prácticas pedagógicas que refirieron los y las docentes de la Facultad de Psicología. La organización de la presentación responde fundamentalmente a la pregunta de investigación acerca qué conocen y qué usos hacen de las TIC en prácticas pedagógicas en el ámbito local.

Solo dos docentes manifestaron no conocer qué es un entorno virtual de enseńanza y aprendizaje. 54 docentes (86\%) daban su correo electrónico a los estudiantes, dos de ellos solían hacerlo pero tuvieron problemas y no lo dan actualmente y siete no están de acuerdo con que los estudiantes tengan su dirección electrónica (Tabla 4). 


\section{Tabla 4}

Los estudiantes disponen de la dirección de correo del docente

\begin{tabular}{lcc}
\hline Los estudiantes tienen la dirección de correo del docente & $\mathrm{f}$ & $\%$ \\
\hline $\begin{array}{l}\text { Sí, todos los años da su dirección de correo a los estudiantes } \\
\text { No, lo hizo alguna vez y tuvo problemas con algún correo recibido }\end{array}$ & 2 & 86 \\
$\begin{array}{l}\text { No, cree que no es pertinente que los alumnos tengan su dirección } \\
\text { de correo }\end{array}$ & 7 & 11 \\
\hline
\end{tabular}

Si bien más de la mitad de los docentes alguna vez cursaron como estudiantes con un entorno virtual (ver Tabla 5), 22 de ellos habían cursado o estaban cursando estudios de posgrado y nunca habían realizado parte de esa formación a través de un aula virtual. De los ocho docentes que nunca habían cursado carreras de posgrado (Tabla 1), tres habían hecho algún tipo de formación utilizando TIC.

\section{Tabla 5}

Docentes que algunas vez cursaron utilizando aula virtual en calidad de estudiantes

\begin{tabular}{lcc}
\hline Alguna vez cursaron utilizando una plataforma virtual & f & $\%$ \\
\hline Sí, tuvo buena/s experiencia/s & 25 & 40 \\
Sí, tuvo alguna/s experiencia/s buena/s y otra/s no tan buenas & 10 & 16 \\
Sí, pero no le gustó la experiencia & 1 & 2 \\
No, nunca cursó con uso de plataforma virtual & 27 & 43 \\
\hline
\end{tabular}

En cuanto al uso de Facebook en docencia, 39 (62\%) de los docentes lo utilizaban o lo habían utilizado (ver Tabla 6). Respecto a las aclaraciones hechas en relación a "Otras respuestas" de la Tabla 6, los docentes dijeron que "la cátedra se comunica con los estudiantes utilizando Facebook"; "a veces utilizo Facebook si los estudiantes lo proponen. En general, me manejo por correo electrónico"; "Esporádicamente algún alumno me localiza por Facebook y solo respondo"; y "Uso compartido por la Cátedra". 
Uso de TIC en prácticas pedagógicas de docentes de la Facultad de Psicología... / Borgobello et al.

\section{Tabla 6}

Utilizan Facebook para la comunicación con los estudiantes

\begin{tabular}{lcc}
\hline Los estudiantes se comunican por Facebook con el docente & $\mathrm{f}$ & $\%$ \\
\hline $\begin{array}{l}\text { Sí, utiliza o utilizó FB en el pasado, pero la experiencia no le resultó } \\
\text { suficientemente positiva }\end{array}$ & 3 & 5 \\
Sí, utiliza FB en la actualidad, pero lo administra un auxiliar & 6 & 10 \\
$\begin{array}{l}\text { Sí, utiliza FB en la actualidad y lo administra personalmente } \\
\text { No, no está de acuerdo con el uso de FB para la comunicación con } \\
\text { los estudiantes }\end{array}$ & 25 & 40 \\
$\begin{array}{l}\text { No, si bien no está en desacuerdo con el uso de FB, nunca lo } \\
\text { consideró como una posibilidad }\end{array}$ & 11 & 18 \\
Otras respuestas & 5 & 8 \\
\hline Total & 63 & 100 \\
\hline
\end{tabular}

Nota. FB = Facebook

Respecto al uso de WhatsApp en la comunicación con los estudiantes (Tabla 7), la gran mayoría de los docentes, 50 (79\%), no lo utilizaban ni utilizaron alguna vez, aunque puede resultar llamativo que 9 (14\%) dijeron usar esta forma de comunicación administrándola personalmente.

Al consultar acerca del uso de plataforma virtual, 39 docentes participantes $(62 \%)$ no recibieron formación alguna en uso de TIC en docencia. Cerca de la mitad de los docentes encuestados nunca utilizaron Moodle u otra plataforma en el dictado de clases (ver Tabla 8). Cuando se les preguntó si conocían que Moodle se encuentra disponible para su uso por parte de todos los docentes de la universidad, 11 (18\%) lo desconocían.

El 44\% de los docentes respondieron que desconocían que en su cátedra se utilice o se haya utilizado plataforma virtual en la enseñanza (Tabla 9). 


\section{Tabla 7}

\section{Utilizan grupos de WhatsApp para la comunicación con los estudiantes}

Los estudiantes se comunican por WhatsApp con el docente

f $\%$

Sí, utiliza o utilizó WhatsApp en el pasado, pero la experiencia no le resultó suficientemente positiva

Sí, utiliza en la actualidad WhatsApp, pero administra el grupo un auxiliar $\begin{array}{lll}2 & 3\end{array}$

Sí, utiliza WhatsApp en la actualidad y lo administra personalmente

No, no está de acuerdo con el uso de WhatsApp para la comunicación con los estudiantes

No, nunca lo consideró como una posibilidad utilizar WhatsApp

\section{Tabla 8}

Utilizan plataforma virtual con los estudiantes

\begin{tabular}{lccc}
\hline Utilizan plataforma virtual con los estudiantes & $\mathrm{f}$ & $\%$ \\
\hline $\begin{array}{l}\text { Sí, ha utilizado PV como docente y la experiencia me pareció positiva } \\
\text { Sí, ha utilizado PV como docente, pero la experiencia no le pareció }\end{array}$ & 28 & 44 \\
$\begin{array}{l}\text { suficientemente positiva como para repetirla } \\
\text { No, nunca utilizó una PV como docente }\end{array}$ & 5 & 8 \\
\hline
\end{tabular}

Nota. $\mathrm{PV}=$ plataforma virtual

\section{Tabla 9}

Otro/s integrantels de la misma cátedra utilizan plataforma virtual con los estudiantes

\begin{tabular}{|c|c|c|}
\hline Otro/s integrante/s de la misma cátedra utilizan PV con los estudiantes & f & $\%$ \\
\hline Sí, alguna vez se ha utilizado PV pero no se utiliza en la actualidad. & 4 & 6 \\
\hline $\begin{array}{l}\text { Sí, uno o algunos de los docentes utilizan PV, pero lo hacen por su propia } \\
\text { cuenta }\end{array}$ & 22 & 35 \\
\hline Sí, se utiliza PV en la cátedra ya que forma parte del proyecto pedagógico & 9 & 14 \\
\hline $\begin{array}{l}\text { No, no tengo conocimiento de uso de PV en mi cátedra por parte de } \\
\text { ningún docente }\end{array}$ & 28 & \\
\hline
\end{tabular}

Nota. $\mathrm{PV}=$ plataforma virtual 
Cabe destacar que 44 (70\%) docentes manifestaron querer tomar un taller sobre Moodle que se ofrecía como parte del presente proyecto de investigación.

Finalmente, mostrando interés por el tema y/o el instrumento, 40 (64\%) agregaron algún comentario que son analizados más abajo en relación a los perfiles grupales del análisis multivariado.

\section{Construcción de perfiles: uso de TIC y docencia}

Desde el objetivo amplio de explorar las características y opiniones de los docentes universitarios de una Facultad de Psicología pública respecto del uso de TIC en las prácticas pedagógicas, se construyeron los perfiles de respondientes a partir de subconjuntos ilustrativos en clusters o grupos. Los clusters ilustran las relaciones entre variables para dar lugar a perfiles diferenciales en cuanto a los resultados principales.

Las variables activas seleccionadas opusieron usos de diferentes TIC mostrando tensión entre las respuestas de los individuos. El primer factor, como puede observarse en la Figura 1, opuso a quienes utilizaban plataforma virtual como docentes y habían cursado online como estudiantes con quienes no habían hecho ninguna de ambas cosas. El segundo factor opone quienes utilizaban Facebook y WhatsApp con sus estudiantes y quienes nunca lo habían hecho.

En cuanto al análisis de clusters, se configuraron tres grupos (véase Figura 2). El perfil típico del primer cluster se caracterizó por docentes que no habían realizado ningún curso utilizando entornos virtuales y utilizaban Facebook en la comunicación con los estudiantes. Las características sobresalientes del segundo cluster se centraron en docentes que realizaron algún tipo de formación con uso de aulas digitales, utilizaban entornos virtuales y Facebook en la comunicación con sus estudiantes. Finalmente, el tercero y último de los clusters, agrupó las respuestas de quienes utilizaban WhatsApp, Facebook y entornos virtuales como docentes. 


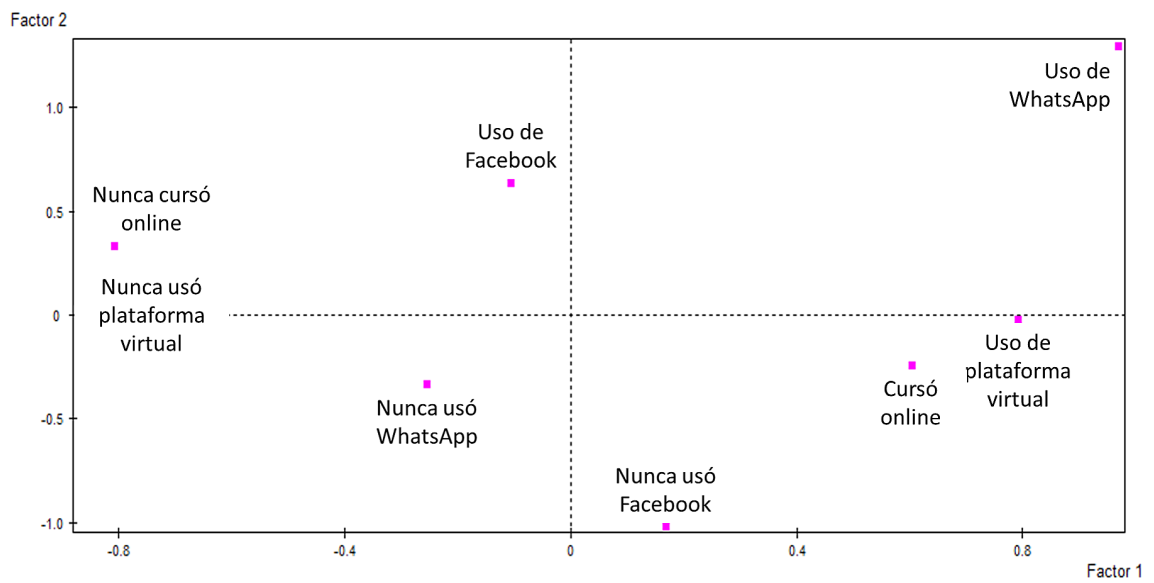

Figura 1. Categorías de las variables activas en los ejes factoriales

Nota. Experiencia en cursado online en calidad de estudiante: Cursó online o Nunca cursó online; en uso de Plataforma Virtual en calidad de docente: Uso de plataforma virtual o Nunca usó plataforma virtual; en uso de WhatsApp como docente: Uso de WhatsApp o Nunca usó WhatsApp; en uso de Facebook como docente: Uso de Facebook o Nunca usó Facebook.

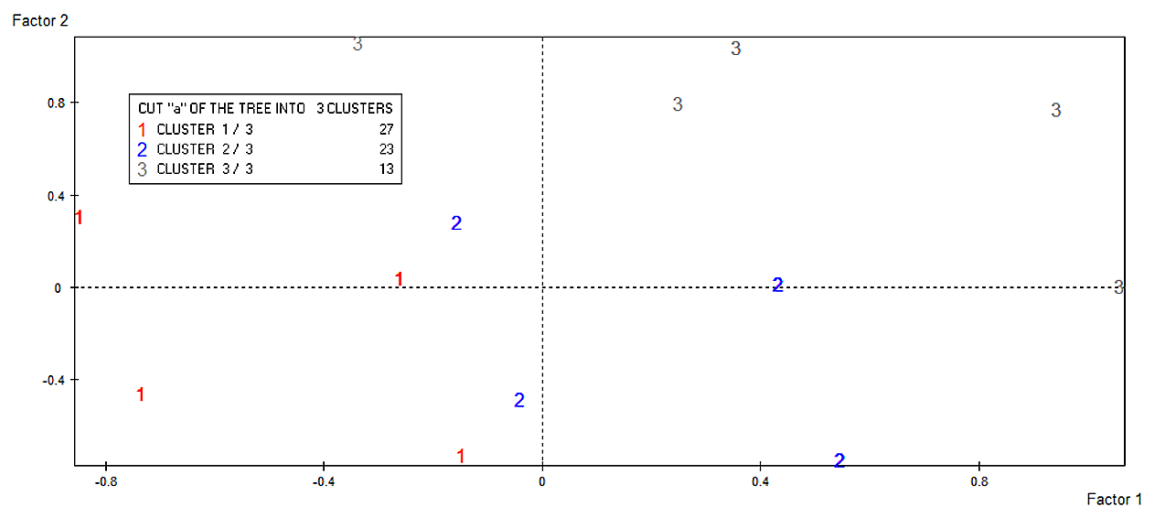

Figura 2. Representación gráfica de los respondientes identificados por los clusters 
A continuación se describen las características principales de cada cluster en función de las variables activas e ilustrativas. Asimismo, cada subconjunto es ilustrado por las críticas pedagógicas, instruccionales e institucionales que los docentes hicieron en relación al uso de TIC en la pregunta abierta (véase Apéndice 1, pregunta 22). Si bien las respuestas fueron variadas, pueden distinguirse características particulares en relación a cada uno de los clusters.

\section{Cluster 1. No a casi todo...}

El primer grupo (integrado por 27 personas, es decir, el $43 \%$ de los participantes) se caracterizó por no utilizar aula virtual como docente, ni WhatsApp, no tener formación específica para utilizar TIC como docente, ni haber cursado como estudiante en un entorno virtual. Se destaca en este grupo un interés por la Psicología Clínica y ninguno de ellos pertenece al Área Socio-Educativa del Plan de Estudios de la Facultad.

Los 10 casos más representativos del cluster 1 (ver Tabla 10) son homogéneos en sus características: todos ellos usaban Facebook con los estudiantes, pero ninguno había cursado online como estudiante, ni había usado plataforma o WhatsApp.

Fueron cinco los comentarios a la pregunta final optativa del cuestionario de los diez casos más representativos del primer cluster. En coherencia con lo anteriormente expuesto, los mismos se enfocaron en exponer las dificultades políticas, técnicas, organizacionales y de formación de recursos humanos (ver Tabla 11).

\section{Cluster 2. Si a las TIC, pero con críticas}

El segundo grupo (conformado por 23 docentes, el 36\% de la muestra) se caracteriza por haber utilizado aula virtual en docencia con experiencias tanto positivas como negativas, pero, a la vez, no haber utilizado WhatsApp con los estudiantes. Consideraban, en general, que el uso de una plataforma virtual es parte de un proyecto pedagógico. 
Estos docentes habían cursado, asimismo, como estudiantes en entornos digitales.

En cuanto a los casos más representativos del cluster 2 (Tabla 10), todos ellos habían cursado como estudiantes online, usaban plataforma con sus estudiantes, casi todos utilizaban Facebook, pero ninguno usaba WhatsApp. En relación a las ocho respuestas abiertas optativas de estas personas, se caracterizaron por un amplio conocimiento del uso de entornos virtuales en el sistema universitario local, lo que les permitió una mirada crítica que reconoce ventajas y desventajas. Destacaron, por ejemplo, la cercanía, la comunicación y la interacción que pueden lograrse con los estudiantes en TIC específicamente diseñadas para ello. Señalaron, asimismo, dificultades técnicas con las que se habían encontrado y la necesidad de soporte institucional al desarrollo de trabajos pedagógicos con TIC (ver Tabla 11).

\section{Tabla 10}

Características de los diez casos más representativos de cada cluster

\begin{tabular}{lccc}
\hline & Cluster 1 & Cluster 2 & Cluster 3 \\
& $\mathrm{f}$ & $\mathrm{f}$ & $\mathrm{F}$ \\
\hline Cursé online & - & 10 & 6 \\
Nunca cursé online & 10 & - & 4 \\
Uso Facebook con los estudiantes & 10 & 9 & 9 \\
No Facebook con los estudiantes & - & 1 & 1 \\
Uso WhatsApp con los estudiantes & - & - & 10 \\
No WhatsApp con los estudiantes & 10 & 10 & - \\
Uso plataforma virtual como docente & - & 10 & 8 \\
No plataforma virtual como docente & 10 & - & 2 \\
\hline
\end{tabular}

Nota. PV = Plataforma Virtual 
Uso de TIC en prácticas pedagógicas de docentes de la Facultad de Psicología... / Borgobello et al.

\section{Tabla 11}

\section{Respuestas abiertas pertenecientes a los diez casos más representativos de cada cluster}

Grupo Respuestas abiertas

Cluster 1 Creo que son herramientas sumamente valiosas que nos acercan a los nuevos modos de enseñar y aprender de esta época. Desconocía que la UNR tenía estos espacios virtuales desde 2001, creo que no fueron correctamente difundidos. Es importante que los docentes nos capacitemos en estas herramientas.

Una de las mayores dificultades para el uso, creo yo es el número, la cantidad de alumnos, la cantidad de integrantes de una cátedra y sus relaciones con estas tecnologías, la disponibilidad de la universidad es poco fiable, en la Facultad de Psicología prácticamente no es posible conectarse a internet, no hay wifi provisto por la institución o funciona muy, muy, muy mal.

Utilizo grupos de Facebook para las dos materias que dicto y me resulta una experiencia sumamente positiva. En una de las cátedras, utilizamos la plataforma virtual de la Facultad de Psicología, pero no la administro yo. Lo que hacemos es subir archivos de clases teóricas con imágenes para los alumnos, pero suele tener problemas la plataforma, por lo cual, también difundimos los archivos a través de los grupos de Facebook de cada comisión.

$\mathrm{Si}$ es de interés socializar el uso de TIC en la comunidad docente sería pertinente organizar alguna capacitación y difusión al respecto.

Por la cantidad de paros que interrumpían el cursado fue un gran recurso poder valerme de los mails y el grupo de Facebook para comunicarme con los estudiantes. Realmente deseaba poder utilizar la plataforma virtual de la Facultad pero por cuestiones de mi cargo no tengo todavía acceso a la misma.
Síntesis de las respuestas en relación con las categorías de análisis

Valoración de aspectos negativos respecto al uso de TIC: críticas en relación a dificultades tecnológicas y de recursos humanos. Plataforma virtual:

- No se utiliza

- No se administra

- No se tiene acceso

- Tiene problemas técnicos

- Falta de capacitación

- Falta de difusión

Usan Facebook 
Grupo Respuestas abiertas
Síntesis de las respuestas en relación con las categorías de análisis
Cluster 2 Al inicio tenía prejuicios, pero todos los medios informáticos que utilicé, Facebook, mail, página, plataforma virtual, etc. tuvieron como resultado mayor compromiso y participación de los estudiantes. Por otro lado, permite resolver problemas cotidianos rápidamente, como por ejemplo distribución de material bibliográfico o seguimiento personalizado de trabajos escritos de los estudiantes. Finalmente, como docente me permite un trato más personalizado y cotidiano con los estudiantes. La única desventaja que encuentro es que el trabajo para el docente, si bien se torna más placentero y cercano por el trato cotidiano con los estudiantes, se incrementa y eso no es pago y por otro lado es invisible para la Facultad como hora de trabajo ya que en personal no se enteran que trabajo esa cantidad de horas extras. En síntesis en lo pedagógico aporta muchísimo, pero es trabajo no pago.

Considero que constituye una herramienta interactiva que puede resultar de utilidad como complemento de las estrategias más tradicionales de enseñanza.

El uso de TIC me parece muy positivo para el proceso de enseńanza-aprendizaje, solo que en ocasiones requiere de demasiado tiempo. En mi caso particular lo empleo como un complemento de las clases presenciales, sobre todo a modo de vía de comunicación y de descarga de bibliografía y otros materiales.

La plataforma de la Facultad no funciona correctamente y eso dificulta nuestras tareas con los estudiantes. Usamos la plataforma cuando funcionaba. Este año nos ha resultado imposible tener acceso adecuado a ella. Todos los docentes manejan Moodle.

Considero tal uso, solo un recurso más entre muchos posibles. Viabiliza la comunicación. No es el soporte básico de la enseñanza.

Las posibilidades del intercambio virtual son muchas pero no se dispone de recursos ni soportes, Facebook por lejos, es más ágil y atractivo (lamentablemente). Lo solucionaríamos con más presupuesto, pero no hay.

Mis alumnos tuvieron problemas para poder ingresar a la plataforma de la UNR cuando la utilicé. Me parece que ayudaría que hubiera en cada unidad académica o en la universidad personal a quien se puedan dirigir docentes o estudiantes cuando tienen estos problemas.
Aspectos positivos del uso de TIC:

+ Diversidad de experiencias con TIC

+ Complemento con clases tradicionales

+ Descarga de materiales

+ Aporte a lo pedagógico

+ Compromiso y

participación estudiantil

+ Uso placentero y cercano

+ Mejora de la

comunicación

Aspectos negativos en

relación al uso de TIC:

- Se necesitaría más personal especializado

- Es trabajo no pago

- No es el soporte básico de la enseñanza.

- No funciona

correctamente por

problemas técnicos

- Requiere tiempo 
Uso de TIC en prácticas pedagógicas de docentes de la Facultad de Psicología... / Borgobello et al.

Grupo Respuestas abiertas

Síntesis de las respuestas en relación con las categorías de análisis

Cluster 3 Me parece interesante y motivadora toda iniciativa tendiente a ampliar y mejorar la comunicación y el intercambio entre docentes, auxiliares y alumnos.

Alta valoración del uso de TIC sin críticas:

Creo que los entornos virtuales enriquecen la - Mejora la comunicación y enseñanza universitaria facilitando tanto el acceso a materiales de uso áulico como la comunicación (fundamentalmente en grupos numerosos).

Me parece muy buena idea utilizar las TIC en la el intercambio - Enriquece la enseñanza

- Supera modelos tradicionales enseñanza pero necesitaría aprender a hacerlo.

Me parece interesante porque no se puede sostener el proceso de enseñanzalaprendizaje con las modalidades tradicionales de transmisión.

Me parece de importancia que se difunda su uso y en particular que se brinde formación gratuita para potenciar el uso que algunos docentes ya estamos haciendo en forma incipiente.

Nota. Las cursivas son de elaboración propia.

Cluster 3. No solo plataforma, también teléfono...

Este grupo (de 13 personas, 21\% de los participantes) se caracteriza por haber tenido experiencias positivas en el uso de entornos virtuales en la enseñanza, pero además, utilizaban WhatsApp en la comunicación con los estudiantes y, generalmente, lo administraban ellos mismos.

En el cluster 3 (Tabla 10), se observa en los diez casos más representativos homogeneidad únicamente en el uso WhatsApp, sin embargo, la mayoría usaban plataforma y Facebook. En cuanto a las cinco respuestas abiertas, se caracterizan por tener alta valoración del uso de TIC en educación superior sin mencionar las dificultades institucionales existentes descritas por otros docentes (Tabla 11). Cabe señalar que en este grupo tampoco se encuentran reflexiones críticas sobre la propia práctica pedagógica con TIC. 


\section{Discusión}

Los encuestados fueron docentes de una Facultad de Psicología pública del centro de Argentina. Las respuestas obtenidas (63) correspondían al $14 \%$ de la población en estudio. No obstante, casi la mitad de los respondientes correspondían a la máxima jerarquía de las cátedras - Titulares, Asociados y Adjuntos- y solo un tercio del total contaba con cargos de dedicación simple — siendo que en la Facultad es la más frecuente de las dedicaciones.

Un dato asociado a destacar es que en la muestra se encontró un alto grado de respondientes con formación de posgrado. Esto puede estar asociado a la jerarquía de los respondientes y a un número importante de docentes del departamento del área de investigaciones. Esta caracterización debiera enmarcar la lectura de los datos.

En cuanto al conocimiento, usos y opiniones sobre TIC, casi todos los docentes conocían qué es un entorno virtual de enseñanza y aprendizaje y la inmensa mayoría utilizaba habitualmente correo electrónico en la comunicación con los estudiantes. Cabe destacar que dos de ellos solían hacerlo, pero tuvieron inconvenientes en el uso y actualmente no lo utilizarían, y siete docentes (11\%) de la muestra consideraban inadecuado que los estudiantes tuvieran su dirección.

Un dato que ilustra características del contexto en cuanto al uso de entornos virtuales es que casi la mitad de los respondientes, aun habiendo hecho carreras de posgrado, nunca cursaron en forma virtual o bimodal — siendo que su uso en posgrado es aún más frecuente que en el grado en el escenario local (Copertari et al., 2015). En cuanto a quienes cursaron como estudiantes con estas mediaciones — virtual o bimodal—, solo uno de ellos manifestó no haber tenido buena experiencia, los demás tuvieron solo buenas o, en su defecto, buenas y no tan buenas experiencias.

En relación al uso de Facebook en docencia, algo más de un tercio de los docentes manifestaron que nunca utilizaron Facebook con los estudiantes, ya sea porque no estaban de acuerdo con ello o nunca lo consideraron una posibilidad. Sin embargo, tal como reportan 
Fernández-Zalazar et al. (2015, 2016) y Fernández-Zalazar y Neri (2015) de la Universidad de Buenos Aires (UBA), su uso es frecuente en una población similar en estudio - en nuestro caso, más de la mitad de los docentes manifestaron haberlo utilizado para comunicarse con los estudiantes. Los autores hallaron escasez de transferencia de habilidades y estraen el uso de TIC desde lo cotidiano al ámbito educativo. Esto se vería reflejado en el uso habitual de recursos digitales impulsados por el mercado - como las redes sociales- y, por otro lado, la escasa implementación de entornos virtuales —especialmente diseñados para la interacción en situaciones de enseñanza y aprendizaje. Solo uno de los docentes encuestados en este estudio comentó que utilizaba Facebook en forma esporádica al ser localizado por este medio por alguno de los estudiantes, limitándose únicamente a responder. En otro caso, la cátedra en su conjunto utilizaba esta red social como parte de su estrategia pedagógica de comunicación e intercambio de archivos.

$\mathrm{Al}$ igual que en los estudios reportados en el contexto de la UBA, la mayor parte de los docentes consultados reportaron no haber recibido formación alguna en uso de TIC en docencia.

Se destaca en los resultados el amplio número de docentes que no estaban de acuerdo en utilizar WhatsApp en la comunicación con los estudiantes. El uso de esta forma de comunicación en el ámbito académico formal no pareciera ser un medio habitual, por lo que sería interesante ahondar en investigaciones futuras los casos en los que se utiliza.

La inmensa mayoría de los encuestados conocían la existencia del campus virtual disponible para todos los docentes en el ámbito de la universidad, pero cerca de la mitad nunca habían utilizado este tipo de medios en las prácticas pedagógicas. Cinco de los docentes que sí habían utilizado plataforma virtual con los estudiantes, no repetirían la experiencia dado que no la consideraron suficientemente positiva. Tal como se destaca en este estudio, parte de esa experiencia negativa puede estar asociada a la escasez de apoyo técnico que los mismos docentes reclamaron.

$\mathrm{El}$ análisis del contexto muestra una universidad en el que predominan modelos clásicos de enseńanza y aprendizaje (García-Aretio, 2017) 
con una tímida tendencia a la bimodalidad (Villar, 2017). Martín y Tourón (2017) visibilizan que las universidades no han sido suficientemente ágiles y efectivas en la integración de TIC en las dinámicas pedagógicas. Por un lado, se observó en el presente estudio que los docentes realizaban reclamos a la institución en relación a mayor apoyo de diversos tipos -técnico, formación específica, entre otras- para la implementación de TIC y, por el otro, Pierella (2014) mostró que los estudiantes reclamaron mayor utilización de TIC en el medio universitario que se reconoce como insuficiente (Copertari et al., 2011, 2015).

Puede remarcarse que los docentes suelen utilizar Moodle desde intereses particulares relativos a la docencia — que en la mayoría de los casos reconoce un mayor trabajo que el dictado de clases tradicionales- y no como parte de proyectos pedagógicos que involucren a cátedras o departamentos de docencia en su conjunto. Este dato ilustra, al igual que en Borgobello et al. (2016) este incipiente uso de medios de comunicación digitales mencionado en el contexto local. En este sentido, FernándezZalazar et al. $(2015,2016)$ muestran uso habitual de TIC en el contexto doméstico no generalizado hacia prácticas educativas formales.

A pesar de lo dicho, cierta carencia en la formación específica en uso de TIC, escasez de proyectos pedagógicos conjuntos que contemplen estas mediaciones, los docentes que respondieron al cuestionario, manifestaron interés en tomar un taller sobre Moodle que se ofrecía como parte del proyecto de investigación. Esto puede deberse, entre otras posibilidades, a que quienes respondieron hayan estado interesados en el tema en estudio o, a partir del estudio, se interesaron en el tema. Asimismo, y en sintonía con lo expresado, en los datos nos encontramos con un número importante de respuestas en las que se narran aspectos específicos del tema en estudio en un espacio que era de llenado optativo, mostrando así interés por opinar sobre el tema.

Finalmente, en el último de los análisis realizados, se construyeron tres perfiles de respondientes o clusters a partir del análisis multivariado de datos. Una conclusión que llama la atención es que la edad, cargo y dedicación docente no parecen haber determinado características propias de los grupos aunque, por ejemplo, es habitual leer sobre el concepto 
Uso de TIC en prácticas pedagógicas de docentes de la Facultad de Psicología... / Borgobello et al.

de nativos digitales, quienes pertenecerían a una generación joven con alto uso de TIC. En la muestra, los docentes más jóvenes, quienes serían los usuarios "naturales" de TIC, no muestran predominancia por sobre los otros docentes ya que la edad y la jerarquía en docencia no aparecen como variables significativas en la construcción de los datos.

El primer perfil de docentes construido, el más homogéneo y al que denominamos "No a casi todo", se caracterizó por el escaso uso de TIC en la vida académica. Es decir, no usaban espacios virtuales formales en la docencia, ni WhatsApp, ni tenían al momento de responder el cuestionario formación específica para utilizar TIC como docentes, ni habían cursado como estudiantes en forma virtual o bimodal. Se destacó en este grupo un interés por la Psicología Clínica, coherente con el perfil de graduado de la Facultad que es fundamentalmente profesionalista y clínico (Klappenbach, 2015). Cabe señalar que en los diez casos más representativos del cluster usaban Facebook con los estudiantes, en sintonía con lo expresado por Fernández-Zalazar et al. $(2015,2016)$ en relación a datos provenientes de otra universidad argentina. En cuanto a los comentarios a la pregunta final optativa del cuestionario de estos diez casos, se enfocaron en exponer dificultades políticas, técnicas, organizacionales y de formación de recursos humanos con cierto grado de desconocimiento de aspectos relativos a diseños tecnopedagógicos en el sentido de Turpo (2010, 2014).

El segundo grupo o cluster, al que denominamos "Sí a las TIC, pero con críticas”, se caracterizó por haber utilizado aula virtual en docencia manifestando haber tenido experiencias tanto positivas como no tan positivas y nunca haber utilizado WhatsApp con los estudiantes -este dato fue homogéneo en los diez casos más representativos del perfil. En este grupo se destacó la idea del uso de plataforma virtual como parte de proyectos pedagógicos superadores de la individualidad del docente y su grupo-clase con perspectivas críticas. Por otro lado, mostrando interés en la implementación de TIC y en el uso personal de las mismas, habían utilizado entornos digitales en calidad de estudiantes.

En cuanto a los diez casos más representativos del segundo grupo, todos ellos habían cursado online como estudiantes, habían utilizado 
plataforma como docentes y casi todos usaban Facebook también. Como rasgo distintivo que lo diferencia del tercer cluster, ninguno de ellos había utilizado WhatsApp para la comunicación con los estudiantes. En relación a las respuestas optativas de este grupo, se caracterizaron por experiencia y formación específica en el uso de entornos virtuales en la universidad, algo que permitió una mirada crítica, se podría decir, con sentido tecnopedagógico (Turpo, 2014), reconociendo ventajas y desventajas en la bimodalidad. Ejemplos de esta mirada son las menciones a la cercanía, fluidez en la interacción y comunicación que permiten estas mediaciones específicamente diseñadas para la enseñanza y el aprendizaje. Estos reconocimientos no resultan ingenuos ya que los docentes del grupo remarcaron dificultades técnicas con las que efectivamente se han encontrado en sus experiencias y la necesidad de mayor soporte institucional para el trabajo con TIC en docencia universitaria.

El tercer cluster fue denominado por el equipo de investigación "No solo plataforma, también teléfono..." debido a que se trata de un grupo —el más reducido en número de casos - que reconoce haber tenido experiencias positivas en el uso de aulas virtuales y que, además, usaban WhatsApp al comunicarse con los estudiantes. De hecho, en los diez casos más representativos de este perfil, observamos homogeneidad únicamente en el uso de WhatsApp, de todos modos, la mayoría de ellos utilizaban, además, plataforma virtual y Facebook. En cuanto a las respuestas abiertas optativas del cuestionario, mostraron una valoración altamente positiva del uso de TIC en educación superior sin mencionar en su mayoría dificultades ni institucionales ni pedagógicas en su implementación concreta.

Los diversos análisis aquí presentados muestran el reclamo por la centralidad del docente en las propuestas educativas (Maggio, 2016). Es decir, no se trataría solo de la disponibilidad de TIC en la enseńanza de nivel universitario sino de comprender de qué modos se utilizan en la actualidad y desde qué concepciones tecnopedagógicas (Turpo, 2014).

A lo largo del escrito puede percibirse la necesidad de articulación entre actores de la universidad como docentes, gestores, estudiantes y técnicos centrándose en las prácticas pedagógicas concretas. De acuerdo a las narraciones de los docentes, la incorporación en proyectos 
pedagógicos con TIC a nivel local parece darse en voluntades individuales aún más que en proyectos político-institucionales.

El desafío, según Lipsman (2016), parece radicar en imaginar la formación que queremos en una universidad pública, comprometida social, política y culturalmente con la democratización de la enseñanza desde principios éticos relacionados con los modos de acceso, producción y circulación de conocimiento. Estos desafíos son posibles de pensar sosteniendo lo mejor de las tradiciones pedagógicas y recreando propuestas formativas acordes a la contemporaneidad para perdurar en el tiempo.

Este trabajo enfocó en las particularidades respecto al uso de TIC de los y las docentes en el contexto en estudio. La recolección de datos fue realizada a partir de un cuestionario autoadministrado online, de escasa utilización a nivel local. Puede entenderse este aspecto como una limitación del estudio presentado (Pozzo et al., 2018). Estudios posteriores tendrían que abordar la relación específica entre prácticas pedagógicas y uso de TIC tal como las entienden docentes y estudiantes del ámbito local a partir de instrumentos que permitan mayor profundidad en la indagación, reconociendo que el contexto condiciona las estrategias tecnopedagógicas, tal como lo plantean Turpo (2014) y González-Fernández (2018).

La generación de conocimiento en torno a la problemática de incorporación de TIC en el ámbito universitario tiene diversas repercusiones prácticas. Este tipo de datos, permite visualizar la escasa virtualización de la enseńanza universitaria local, tal como lo sostienen Copertari et al. (2011, 2015). A partir de ello, contribuye al reconocimiento reflexivo y situado que posibilita ampliar la virtualización reconociendo la realidad tecnopedagógica. Si bien la tendencia en Argentina se da hacia la bimodalidad (Villar, 2017), el sistema universitario se avizora aún como fuertemente presencial.

Es a partir de cuestionamientos colectivos, en trabajos cooperativos y colaborativos que podría la universidad superar esta visión de una institución que parece estar más ligada a lo tradicional y conservador que a visiones pedagógicas más contemporáneas y vinculadas al uso de TIC en la mediación pedagógica. 


\section{Referencias bibliográficas}

Aluja-Banet, T. \& Morineau, A. (1999). Aprender de los datos: el análisis de componentes principales. Una aproximación desde el data mining. Barcelona: EUB.

Boelens, R., De-Wever, B. \& Voet, M. (2017). Four key challenges to the design of blended learning: A systematic literature review. Educational Research Review, 22(1), 1-18. https://dx.doi. org/10.1590/S1517-9702201606143478

Borgobello, A. \& Roselli, N. D. (2016). Rendimiento académico e interacción sociocognitiva de estudiantes en un entorno virtual. Educação e Pesquisa, 42(2), 359-374.

Borgobello, A., Sartori, M. \& Sanjurjo, L. (2018). Concepciones de docentes sobre los estudiantes y sus prácticas pedagógicas. Educación y Educadores, 21(1), 27-48. https://doi.org/10.5294/ edu.2018.21.1.2

Borgobello, A., Sartori, M. \& Roselli, N. (2016). ¿Cómo interactuamos aquí y allá? Análisis de expresiones verbales en una clase presencial y otra virtual a partir de dos sistemas de codificación diferentes. Revista de la educación superior, 45(179), 95-110. https://dx.doi.org/10.1016/j.resu.2016.06.003

Copertari, S., Sgreccia, N. y Segura, M.L. (2011). Políticas universitarias, gestión y formación docente en educación a distancia. Hacia una pedagogía de la virtualización. Revista de Educación a Distancia, 27(1), 1-16. http://www.um.es/ead/red/27

Copertari, S., Sgreccia, N., Rosales, M., Fantasía, Y., Aita, G. \& Segura, M.L. (2015). Comunidades virtuales y formación docente en la UNR. En C. Pairoba (coord.), Ciencia y tecnología 2015: divulgación de la producción científica y tecnológica de la UNR (pp. 765-769). Rosario: UNR Editora.

Eagleton, S. (2017). Designing blended learning interventions for the 21st century student. Advances in Physiology Education, 41(2), 203-211. https://doi.org/10.1152/advan.00149.2016 
Fernández-Zalazar, D. y Neri, C. (2015). Apuntes para la revisión teórica de las TIC en el ámbito de la educación superior. RED. Revista de Educación a Distancia, 47(3), 1-8. http://www.um.es/ $\mathrm{ead} / \mathrm{red} / 47$

Fernández-Zalazar, D., Jofre, C. \& Soto, R. (2016). Prácticas docentes y TIC en el nivel superior. Anuario de Investigaciones, 23(1), 105-113.

Fernández-Zalazar, D., Jofre, C., Pisani, P. A. \& Ciacciulli, S. (2015). Aproximación a los usos de las TIC y las prácticas de enseñanza en docentes universitarios de psicología. Anuario de Investigaciones, 22(1), 143-151.

García-Aretio, L. (2017). Educación a distancia y virtual: calidad, disrupción, aprendizajes adaptativo y móvil. RIED. Revista Iberoamericana de Educación a Distancia, 20(2), 9-25. https://doi. org/10.5944/ried.20.2.18737

González-Fernández, M. O. (2018). Percepción del desempeño docente-estudiante en la modalidad mixta desde una mirada ecosistémica / Perception of teacher-student performance in the mixed mode from an ecosystemic perspective. Revista Iberoamericana para la Investigación y el Desarrollo Educativo, 8(16), 346-370. https://doi.org/10.23913/ride.v8i16.346

Klappenbach, H. (2015). La formación universitaria en psicología en Argentina: perspectivas actuales y desafíos a la luz de la historia. Universitas Psychologica, 14(3), 937-960. https://doi. org/10.11144/Javeriana.upsy14-3.fupa

Lipsman, M. (2016). La innovación con tecnologías en las propuestas de enseńanza de grado. En M. Insaurralde (comp.). La enseñanza en la educación superior. Investigaciones, experiencias y desafios (pp. 139-156). Buenos Aires: Noveduc.

Maggio, M. (2016). Enriquecer la enseñanza: los ambientes con alta disposición tecnológica como oportunidad. Buenos Aires: Paidós.

Martín R., D. \& Tourón, J. (2017). El enfoque flipped learning en estudios de magisterio: percepción de los alumnos. RIED. 
Revista Iberoamericana de Educación a Distancia, 20(2), 187211. https://doi.org/10.5944/ried.20.2.17704

Moscoloni, N. (2005). Las nubes de datos. Métodos para analizar la complejidad. UNR Editora.

Parra-Olivares, J. E. (1996). Modelo de análisis de correspondencias múltiples. Revista de Ciencias Sociales, 2(2), 183-196.

Pierella, M.P. (2014). La autoridad de los profesores desde la perspectiva estudiantil. Revista Brasileira de Educação, 19(59), 893-912. https://doi.org/10.1590/S1413-24782014000900005

Pozzo, M.I., Borgobello, A. \& Pierella, M.P. (2018, en prensa). Uso de cuestionarios en investigaciones sobre universidad: análisis de experiencias desde una perspectiva situada. Revista Latinoamericana de Metodología en Ciencias Sociales, 8(2).

Turpo, O.W. (2010). Contexto y desarrollo de la modalidad educativa blended learning en el sistema universitario iberoamericano. Revista Mexicana de Investigación Educativa, 15, 345-370.

Turpo, O. W. (2014). Perspectiva de la convergencia pedagógica y tecnológica en la modalidad blended learning. Educación, 23(44), 67-87.

Twining, P., Heller, R. S., Nussbaum, M. \& Tsai, C.-C. (2017). Some guidance on conducting and reporting qualitative studies. Computers \& Education, 106, A1-A9. https://doi.org/10.1016/ j.compedu.2016.12.002

Villar, A. (2017). Hacia la universidad bimodal: dimensiones de un proceso incipiente. En M.E. Collebechi y F. Gobato (comps.), Formar en el horizonte digital (pp. 129-136). Bernal: Universidad Virtual de Quilmes. Recuperado de: http://libros.uvq.edu.ar/

Recibido: 27 de diciembre, 2017

Revisado: 14 de agosto, 2018 Aceptado: 17 de setiembre, 2018 
Uso de TIC en prácticas pedagógicas de docentes de la Facultad de Psicología... / Borgobello et al.

\section{Apéndice 1}

Este Cuestionario está dirigido a docentes universitariosy forma parte del Proyecto de Investigación PSI334 "Los entornos virtuales en la enseñanza universitaria" aprobado por las instancias correspondientes de acuerdo a las regulaciones vigentes en la Universidad ------- y radicado en la Facultad de Psicología.

Las preguntas refieren a intereses generales y uso de TIC (tecnologías de la información y la comunicación) en docencia universitaria.

Sobre el final del cuestionario encontrará un espacio abierto para opinar sobre los temas indagados o ampliar cualquiera de sus respuestas.

TODAS SUS RESPUESTAS SON ANÓNIMAS

Quienes participen del proyecto voluntariamente podrán retirarse en el momento que lo deseen durante el desarrollo de la investigación sin que ello ocasione perjuicio alguno.

Recuerde que las respuestas se basan en su experiencia y que no existen respuestas correctas o incorrectas.

Muchas gracias por su colaboración, la misma tomará unos pocos minutos.

Equipo de investigación PSI334:

- Acepto participar

1. Título de Grado:

2. Título Máximo de Posgrado (señale si está en curso o finalizado) :

3. Edad:

4. Tipo de cargo docente (marcar todos los que posee en la universidad):

- Auxiliar de segunda

- Auxiliar de Primera

- Adscripto/a

- Jefe de trabajos prácticos

- Adjunto

- Asociado

- Titular de Cátedra

5. Dedicación en docencia (sumando todos los cargos):

- Simple

- Semi-exclusiva

- Exclusiva 
6. Antigüedad en la docencia en años (desde Auxiliar de Segunda a la actualidad) :

7. Año/s de cursado en el que se encuentra su/s cátedra/s (puede indicar más de una opción si trabaja en más de una materia):

- Primer Año

- Segundo Año

- Tercer Año

- Cuarto Año

- Quinto Año

- Sexto Año

- Profesorado en Psicología

8. Departamento en el que se inscribe su materia de acuerdo al Plan de Estudios vigente (puede indicar más de una opción si trabaja en más de una materia):

- Primer Año

- Área Social y Comunitaria

- Área de Psicoanálisis

- Área Histórico-Epistemológica

- Área Socio-Educativa

- Área Investigaciones

- Área de Clínicas

- Área Psicología del Desarrollo y Psicoterapias

9. Cantidad de clases semanales que dicta en la universidad:

10. Cantidad estimada de alumnos (sumando los grupos a su cargo este año):

11. ¿Desea hacer alguna aclaración sobre alguna particularidad del dictado de clases a su cargo? Ejemplo: doy teóricos en una materia y prácticos en otra.

12. ¿Qué área disciplinar es de su interés? (puede marcar más de una opción):

- Laboral

- Educativa

- Jurídica/Forense

- Clínica

- Investigación

- Comunitaria 
Uso de TIC en prácticas pedagógicas de docentes de la Facultad de Psicología... / Borgobello et al.

- Filosofía

- Social

- Salud

- Psicoanálisis

- Psicoterapias breves

- Ambiental

- Psicología Positiva

- Sexualidad y género

- Derechos humanos

- Orientación vocacional

- Discapacidad

- Deportes

- Desarrollo

- Neurociencias

- Epistemología

- Historia

- Ética

- Psicología del consumidor

- Familia

- Emergencias, urgencias y desastres

- Psicometría

- Otros:

13. ¿¿Sabe qué es una plataforma, espacio o entorno virtual?

- Sí, conozco de qué se trata

- No, desconozco qué es

14. ¿¿Sabe que la Facultad de Psicología y la Universidad Nacional de Rosario disponen de espacios virtuales (desde 2001) a disposición de los docentes para complementar el dictado de las clases?

- Sí

- No

Las preguntas siguientes se refieren a Plataformas virtuales y otras TIC tales como correo electrónico.

15. Respecto de la comunicación sus alumnos: ¿tienen su dirección de correo electrónico a disposición?

- Sí, todos los años doy mi dirección de correo a los estudiantes 
- No, lo hice alguna vez y tuve problemas con algún correo recibido

- No, creo que no es pertinente que mis alumnos tengan mi dirección de correo

16. ¿Alguna vez cursó (como estudiante de grado o posgrado) con plataforma virtual?

- Sí, tuve buena/s experiencia/s

- Sí, tuve alguna/s experiencia/s buena/s y otra/s no tan buena/s

- Sí, pero no me gustó la experiencia

- No, nunca cursé con uso de plataforma virtual

17. ¿Utiliza o utilizó Facebook para la comunicación con sus estudiantes?

- Sí, utilicé en el pasado, pero la experiencia no me resultó suficientemente positiva

- Sí, utilizo en la actualidad, pero lo administra un auxiliar

- Sí, utilizo en la actualidad y lo administro personalmente

- No, no estoy de acuerdo con el uso de Facebook para la comunicación con los estudiantes

- No, si bien no estoy en desacuerdo con su uso, nunca lo consideré como una posibilidad

18. ¿Utiliza o utilizó grupo de Whatsapp para la comunicación con sus estudiantes?

- Sí, utilicé en el pasado, pero la experiencia no me resultó suficientemente positiva

- Sí, utilizo en la actualidad, pero administra el grupo un auxiliar

- Sí, utilizo en la actualidad y lo administro personalmente

- No, no estoy de acuerdo con el uso de Whatsapp para la comunicación con los estudiantes

- No, nunca lo consideré como una posibilidad

19. ¿Recibió alguna formación específica en el uso de TIC en la enseñanza?

- Sí, hice uno o más cursos

- No, nunca recibí formación específica sobre el tema

20. ¿Utilizó COMO DOCENTE una plataforma virtual?

- Sí, he utilizado como docente y la experiencia me pareció positiva

- Sí, he utilizado como docente, pero la experiencia no me pareció suficientemente positiva como para repetirla 
Uso de TIC en prácticas pedagógicas de docentes de la Facultad de Psicología... / Borgobello et al.

- No, nunca utilicé una plataforma como docente

21. ¿Algún otro/a integrante de su cátedra alguna vez utilizó plataforma virtual (Moodle u otra plataforma virtual de enseñanza y aprendizaje) con los estudiantes? *

- Sí, alguna vez se ha utilizado pero no se utiliza en la actualidad.

- Sí, uno o algunos de los docentes, pero lo hacen por su propia cuenta

- Sí, se utiliza en la cátedra ya que forma parte del proyecto pedagógico

- No, no tengo conocimiento de su uso en mi cátedra por parte de ningún docente

22. Nos gustaría que emita su opinión acerca de alguna de las preguntas del cuestionario o sobre el uso de TIC o entornos virtuales en la enseñanza universitaria. Siéntase libre de opinar abiertamente ya que todo el contenido del cuestionario se analizará de forma anónima y sus comentarios son muy importantes para nuestra investigación:

23. Como parte del Proyecto de investigación, hemos diseñado un taller virtual para docentes de la Facultad que deseen iniciarse en el uso de Moodle (plataforma virtual disponible para los docentes de la Facultad) como complemento del aula tradicional de clases.

- Me gustaría participar del Taller Virtual sobre iniciación en uso de Moodle 